\title{
Tortuous Duplicated Collecting System Complicated by Ureterovesical Junction Compression
}

\author{
Sagar Patel Obafunbi Abimbola Ornob P. Roy \\ McKay Urology, Atrium Health, Charlotte, NC, USA
}

\author{
Key Words \\ Duplex collecting system • Heminephroureterectomy • \\ Ureterovesical junction obstruction • Heminephrectomy • \\ Robotic-assisted laparoscopic surgery
}

\begin{abstract}
Duplex collecting system of the kidney is a common congenital anomaly of the urinary tract and is less reported in the adult population. Rarely, this anomaly can result in ureterovesical junction compression. Herein, we present a case of ureterovesical junction compression occurring in an adult patient with a duplex collecting system and describe the surgical management.

(c) 2020 The Author(s) Published by S. Karger AG, Basel
\end{abstract}

\section{Introduction}

One of the most common anomalies of the urinary tract, duplex collecting system of the kidney has a reported prevalence of 1 in 125 births [1]. Usually, patients with duplicated kidneys and ureters present in childhood with recurrent episodes of urinary tract infections (UTIs) or ureteral obstruction. Additionally, this condition may

\section{KARGER}

Fax +4161306 1234

E-Mail karger@karger.com

www.karger.com
(C) 2020 The Author(s) Upen access

This article is licensed under the Creative Commons AttributionNonCommercial-NoDerivatives 4.0 International License (CC BY NC-ND) (http://www.karger.com/Services/OpenAccessLicense). Usage and distribution for commercial purposes as well as any distribution of modified material requires written permission. be missed during childhood and become symptomatic in adulthood requiring surgical correction. To our knowledge, we report a rare complication of duplicated collecting system - ureterovesical junction (UVJ) compression - and its surgical management.

\section{Case Report}

A 60-year-old male smoker presented to clinic with intermittent right flank pain for several weeks. He denied low back pain, dysuria, or gross hematuria. He has had episodes of UTIs in the past. His past medical history is significant for hypertension, newly diagnosed lymphoma, and duplicated collecting system discovered 1 year ago via computed tomography (CT) scan. His family and social history are non-contributory. Upon diagnosis of duplex collecting system, patient underwent mercaptoacetyltriglycine-3 lasix renal scan to determine renal function and possible obstruction. The scan revealed asymmetric flow with differential kidney function $-63 \%$ flow of the left kidney and $37 \%$ flow of the right kidney - in addition to a large cold defect within the upper right kidney pole. Patient did not follow-up for further evaluation of renal anomaly.

In clinic, he was afebrile with a blood pressure of 178/88 $\mathrm{mmHg}$. His abdomen was unremarkable and no costovertebral angle tenderness was appreciated. Laboratory analysis revealed a white blood cell count of $20,900 / \mu 1$ most likely secondary to underlying malignancy. A urine analysis was significant for trace 


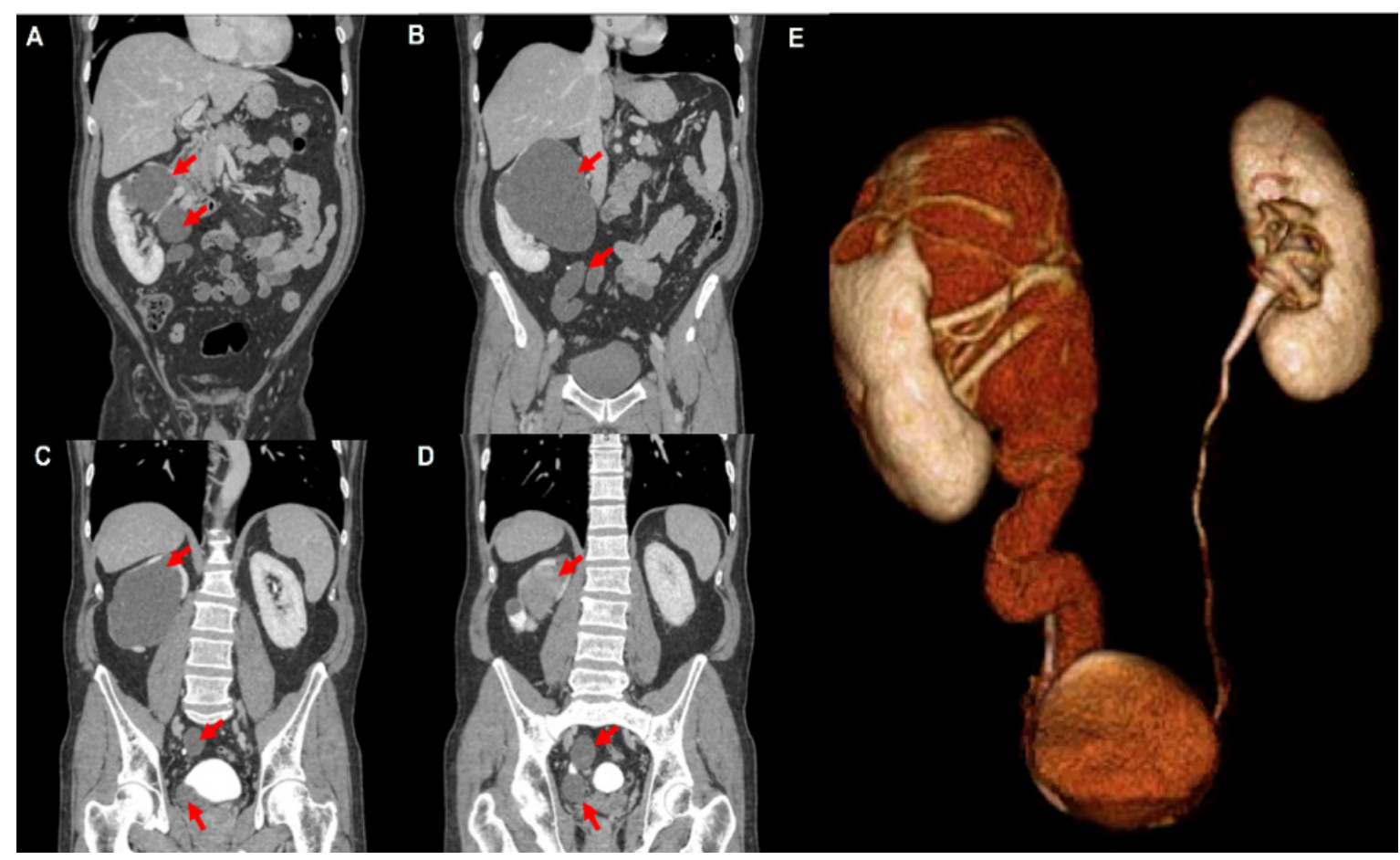

Fig. 1. Serial CT with contrast of abdomen and pelvis. A-D Red arrows indicate upper moiety of the duplicated system; $\mathbf{E}$ Three-dimensional reconstruction of the serial CT shows severe hydroureteronephrosis of the right upper ureter and moiety.

blood and negative for leukocytes. The patient underwent a CT urography of his abdomen and pelvis, which identified severe, tortuous right sided hydroureteronephrosis involving the upper pole moiety of the duplicated kidney. There was no evidence of intra-ureteral obstruction. Lower pole moiety showed normal contrast filling of the renal pelvis and ureter without evidence of dilatation (fig. 1A-D). Reconstructed urography sections suggested ectopic insertion of the right upper ureter at the bladder neck and compression at the UVJ (fig. 1E). The patient underwent robotic right upper pole moiety heminephroureterectomy. Intraoperative retrograde ureteropyelogram showed normal appearing right lower ureter connecting to the lower pole moiety without connection to the duplicated system (fig. 2). During the procedure, the upper moiety and corresponding ureter were removed as well as a duplicated renal artery and vein. There were no intraoperative complications. Postoperatively, the procedure was complicated by a urine leak, which resolved within 2 weeks. Patient was recently seen in office and is doing well.

\section{Discussion}

Duplex collecting system is a common congenital anomaly in which a kidney has two attached collecting systems, each draining to its own ureter - one in the upper pole and the other in the lower pole. During the development of the ureters, the caudal end of the Wolffian duct forms the ureteric bud. The ureteric bud grows cephalad forming the ureter and subsequently divides into several buds creating the renal pelvis and renal calyces; this is known as the upper urinary tract [2]. The ureteric bud fuses with the metanephros to create a normal functioning kidney [3].

There are two anatomic variants of duplex collecting systems: incomplete and complete duplication. Although the embryologic origin of incomplete duplication is not well understood, this anomaly may arise from early branching of the ureteric bud [4]. As a result, only one ureteral orifice is present on the ipsilateral side, and along the midsegment of the ureter, there is a bifurcation point to the duplicated kidney moiety. Alternatively, a complete duplicated collecting system arises from two separate ureteral buds and connects with the metanephros [3]. An incomplete duplex collecting system is 3 times more common than a complete duplication. Duplex collecting 


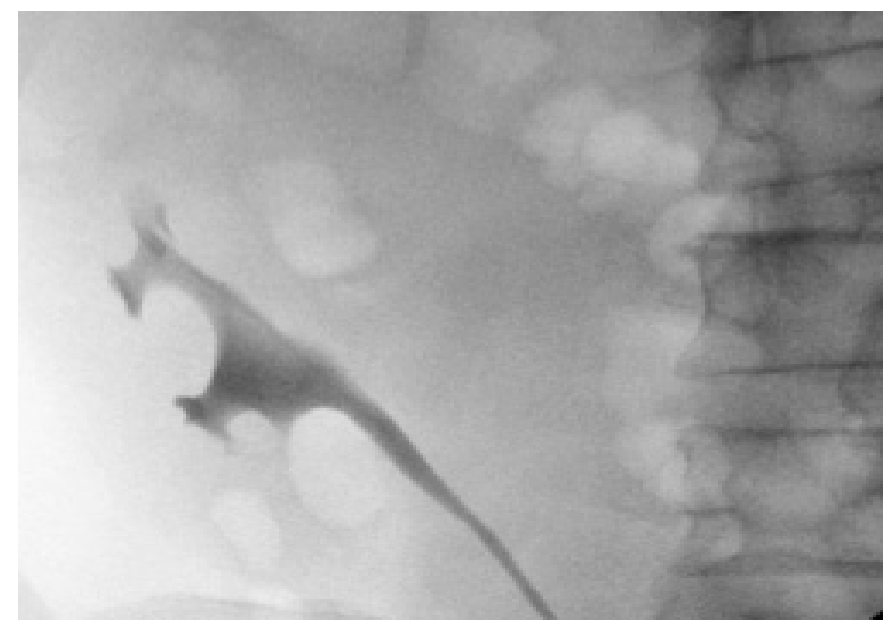

Fig. 2. Retrograde ureteropyelogram shows contrast filling of the right lower moiety and ureter. There is no filling of the upper moiety and corresponding ureter suggesting a complete duplex system.

systems are twice as common in females as in males. Of the reported renal duplication, approximately $17 \%$ patients have bilateral duplicated systems [5].

Clinical presentation of duplicated systems includes hematuria, flank pain, abdominal pain, and recurrent UTIs. Although diagnosis typically occurs during childhood, asymptomatic diagnosis of duplex collecting systems have become more common in the adult population with increasing use of imaging modalities. Furthermore, congenital anomalies can be overlooked in the older population presenting with recurrent UTIs. In a case report, a 40-year-old diabetic female had several episodes of diabetic ketoacidosis secondary to UTIs. Renal imaging showed a duplicated collecting system that predisposed her to these infections and subsequently, hyperglycemic events [6]. Other complications of this anomaly are vesicoureteral reflux, ectopic ureterocele, and rarely ureteropelvic junction (UPJ) obstruction [4, 7]. Ectopic ureteroceles are 8 times more common in females than males whereas UPJ obstruction is more common in males [4]. Severe vesicoureteral reflux can present with hydroureteronephrosis [8]. In this case, evidence of reflux was not seen on the renal scan. To our knowledge, there are no cases of UVJ compression of a completely duplicated collecting system reported. One of the more common obstructive complications of this anomaly is UPJ obstruction because this region is narrow. Typically with UPJ obstruction of a duplicated collecting system, the lower pole moiety is affected [9].
There are ample published studies and case series in the pediatric population about heminephrectomies for duplicated systems. Conversely, there is only a smaller cohort of adult data regarding the surgical management of duplex collecting systems. Interestingly, when operatively managing renal duplication, there are few complications associated with heminephrectomies in the pediatric population compared to the adult population. Clement et al. [10] believe that this discrepancy is attributed to the fewer cases of heminephrectomies in adult patients with duplicated collecting systems and thus less experience operating on this anatomical variant. This procedure in the adult population with duplicated collecting system has a success rate of $96.5 \%$ and a complication rate of $6.3 \%$. Complications include urine leak, infection, hematoma, and blood loss. Furthermore, most cases of renal duplications are corrected laparoscopically [10]. Limited data is available about the robotic heminephrectomies in patients with duplex collecting systems.

Although duplex collecting systems are common congenital anomalies, initial presentation of duplicated systems in adults are less reported than the children. In this case report, we present an uncommon complication of complete collecting system duplication in the adult population that required surgical management.

\section{Acknowledgement}

The authors acknowledge the Atrium Health Department of Radiology. 


\section{References}

1 Wein AJ, Kavoussi LR, Partin AW, Peters CA: Campbell-Walsh Urology, ed 11. Philadelphia, Elsevier, 2016.

2 el-Galley RE, Keane TE: Embryology, anatomy, and surgical applications of the kidney and ureter. Surg Clin North Am 2000;80:381401.

-3 Didier RA, Chow JS, Kwatra NS, Retik AB, Lebowitz RL: The duplicated collecting system of the urinary tract: embryology, imaging appearances and clinical considerations. Pediatr Radiol 2017;47:1526-1538.
4 Fernbach SK, Feinstein KA, Spencer K, Lindstrom CA: Ureteral duplication and its complications. Radiographics 1997;17:109-127.

5 Privett JT, Jeans WD, Roylance J: The incidence and importance of renal duplication. Clin Radiol 1976;27:521-530.

-6 Raja J, Mohareb AM, Bilori B: Recurrent urinary tract infections in an adult with a duplicated renal collecting system. Radiol Case Rep 2016;11:328-331.

-7 Basdas C, Celebi S, Ozaydin S, Karaaslan B, Alim ER, Guvenc U, Sander S: Unusual presentation of duplex kidneys: ureteropelvic junction obstruction. Adv Urol 2016;2016:7960794.
-8 Woodward M, Frank D: Postnatal management of antenatal hydronephrosis. BJU Int 2002;89:149-156.

9 Ulchaker J, Ross J, Alexander F, Kay R: The spectrum of ureteropelvic junction obstructions occurring in duplicated collecting systems. J Pediatr Surg 1996;31:1221-1224.

10 Clement K, Hendry J, Keeley FX, Aboumarzouk OM: Heminephrectomy in adults: a systematic review with cumulative analysis. Urol Int 2018;101:1-6. 